\title{
Energy Levels and Spectral Lines for $n=3$ to $n=3$ Transitions in Mg-Like Tungsten Ions
}

\author{
L.H. HAO AND X.P. KANG* \\ School of Science and Technology, Qiongzhou University, Sanya 572022, Hainan, China
}

(Received April 16, 2015; in final form September 22, 2015)

Energies and transitions for allowed (E1) and forbidden (E2, M1, and M2) lines for low-lying configurations in magnesium-like tungsten $\left(\mathrm{W}^{62+}\right.$ ) are studied using the multiconfiguration Dirac-Hartree-Fock approach. It is shown that the correlations within the $n=3$ complex, quantum electrodynamic and Breit effects are very important for the calculation of fine structure energies. The present results are compared to and agree very well with other theoretical energies, wavelengths, transition probabilities and line strengths previously published for a few lines, and are generally found to be more reliable than previous theoretical predictions. The data for E2, M1, and M2 for transitions between the levels are presented as supplemental material. Results are also compared with other theory, when available.

DOI: 10.12693 /APhysPolA.128.274

PACS: 31.30.Gs, 32.30.-r

\section{Introduction}

Accurate atomic structure and spectral data of highly ionized tungsten (W; $Z=74$ ) ions are of great importance in plasma science fusion reaction, high-energy astrophysics, biomedical applications, and other scientific research fields. The scientific studies and applications of highly ionized ions have been limited because these ions are exiguous on Earth and the data obtained from observing cosmic sources by the relatively high X-ray absorption of Earth's atmosphere are not enough [1]. In addition, forbidden lines play an essential role in plasma diagnostics because the corresponding radiant intensities are often very sensitive to electron density and temperature. Therefore, accurate atomic data including information on atomic transitions for such forbidden lines in various ionization stages of tungsten must be determined with high confidence from theoretical calculations or experimental measurements.

There are not many experimental and theoretical studies in the literature for $n=3$ to 3 transition energies of highly charged tungsten ions. With some experimental data now available, a detailed analysis of extreme ultraviolet (EUV) spectra of highly charged tungsten ions $\mathrm{W}^{54+}-\mathrm{W}^{63+}$ obtained with an electron beam ion trap (EBIT) was presented by Ralchenko et al. [2]. Gillaspy et al. measured the $3 s_{1 / 2}-3 p_{1 / 2}$ and the $3 s_{1 / 2}-3 p_{3 / 2}$ transitions in Na-like ions with $Z \geq 72$, including tungsten, together with the $3 s_{1 / 2}-3 p_{3 / 2}$ transition in $\mathrm{Mg}$ like and the $3 p_{1 / 2}-3 d_{3 / 2}$ transition in Al-like and Si-like ions, at the National Institute of Standards and Technology EBIT facility [3]. The measured $3 s_{1 / 2}-3 p_{3 / 2}$ line positions, however, did not have the accuracies required to differentiate between theories. Clementson and

*corresponding author; e-mail: xpk710@126.com
Beiersdorfer [4] carried out more accurate wavelength measurements of $n=3$ to $n=3$ transitions in K-like $\mathrm{W}^{55+}$ through Ne-like $\mathrm{W}^{64+}$ ions at the electron-beam ion trap facility in Livermore. Recently, Clementson et al. [5] measured the energy of $n=2$ to $n=2,3$ transitions in Ne-like $\mathrm{W}^{64+}$ through Li-like $\mathrm{W}^{71+}$ ions and soft X-ray measurements of $n=3$ to $n=3,4$ transitions in M-shell ions with emphasis on the Ni-like $\mathrm{W}^{46+}$ and Silike $\mathrm{W}^{60+}$ through Na-like $\mathrm{W}^{63+}$ ions at the Livermore EBIT-1 electron-beam ion trap and SuperEBIT electron beam ion traps.

On the theoretical side, numerous papers were studied to the investigation of properties of Mg-like ions; however, only few papers have considered the properties of highly charged ions with nuclear charge $Z=74, \mathrm{~W}^{62+}$. Energies and lifetime values for the $3 s 3 p{ }^{13} P_{J}$ levels evaluated by the multiconfiguration Dirac-Hartree-Fock (MCDHF) approach (Desclaux's code) were given for Mg-like tungsten [6]. The influence of the hyperfine interaction on the energy separation of the $3 s 3 p^{3} P_{0,1}$ levels as a function of nuclear spin $I$ and the atomic number $Z$ was investigated by Marques et al. [6]. Energies and forbidden transition rates between the $3 s^{2}$ and $3 s 3 p{ }^{13} P_{J}$ levels calculated using the MCDHF approach were presented by Zou and Froese Fischer [7] for Mglike tungsten. Energy levels, radiative and autoionization rates for $3 l^{\prime} n l(n=3-13, l \leq n-1), 4 l^{\prime} n l(n=4-7$, $l \leq n-1)$ and $2 p 53 l^{\prime} l^{\prime \prime} n l(n=3-4, l \leq n-1)$ states in large range of highly ionized tungsten ions were computed by Safronova et al. [8, 9]. The energy levels and spectral lines of multiply ionized tungsten atoms, $\mathrm{W}^{2+}$ through $\mathrm{W}^{73+}$, were compiled by Kramida and Shirai [10]. A list of energy levels for $\mathrm{W}^{62+}$ includes only four values $\left(3 s 3 p^{13} P_{J}\right)[10]$. As a result, the radiation on $\mathrm{Mg}$ like tungsten and other tungsten ions have been studied in several previous works both theoretically and experimentally, and several overviews of the existing effort and available data have been given [11-23]. 
The aim of this paper is to produce accurate atomic structure and spectral data for allowed and forbidden transitions of Mg-like tungsten $\left(\mathrm{W}^{62+}\right)$ using the MCDHF approach of the general relativistic atomic structure package, GRASP2K [24]. In a revised version, the calculations not only overcame some limitations on the complexity of the cases that could be executed successfully by GRASP2K but also increased the efficiency and the speed of angular integrations. In the present paper, the MCDHF-calculated the energies and radiative transition (E1) parameters including the wavelengths, transition probabilities and line strengths for $n=3$ to 3 transitions are compared with results from the relativistic many-body perturbation theory (RMBPT) by Safronova et al. [8, 9]. Produced atomic data, which include energy levels, transition rates, oscillator strengths and wavelengths of E1, E2, M1, and M2 transitions, could be used in low density and hot dense plasma diagnostics.

\section{Theoretical method}

The GRASP2K computer package and its revision are based on the MCDHF approach taking into account relativistic and quantum electrodynamic (QED) corrections $[25,26]$.

In this approach, the notion of atomic state function (ASF) is introduced; an ASF $\psi(\gamma P J)$, for a stationary state of an atom, expressed as a linear combination of symmetry-adapted CSFs $\Phi\left(\gamma_{i} P J\right)$, i.e.

$$
\psi(\gamma P J)=\sum_{i}^{N C F} c_{i} \Phi\left(\gamma_{i} P J\right)
$$

where $J$ is the total electronic angular momentum of the state, $\gamma$ represents the electronic configuration and intermediate quantum numbers, $P$ stands for the parity and $\mathrm{NCF}$ is the number of CSFs involved in the expansion. The $c_{i}$ are the so-called mixing coefficients and must follow the normalization condition

$$
\sum_{i}^{N C F} c_{i}^{2}=1
$$

reflecting a part of the orthonormality that is required between CSFs. The set of orbitals that underlie the CSF basis is the active set (AS) and if no restriction is placed on the generation of CSFs, the resulting expansion constitutes the so-called complete active space (CAS).

The multiconfiguration energy functional is based on the Dirac-Coulomb Hamiltonian, namely (in a.u.),

$$
H_{D C}=\sum_{i=1}^{N}\left(c \alpha_{i} p_{i}+\left(\beta_{i}-1\right) c^{2}+V_{i}^{N}\right)+\sum_{i>j}^{N} \frac{1}{r_{i j}}
$$

where $\alpha$ and $\beta$ are $4 \times 4$ Dirac matrices, $p$ is the momentum operator, $V_{i}^{N}$ and $1 / r_{i j}$ are the monopole part of the electron-nucleus and electron-electron interactions, respectively. In the relativistic self-consistent field procedure both the radial parts of the Dirac orbitals and the expansion coefficients are optimized to self-consistency.

The contributions from the transverse photon (Breit) interaction, QED and finite nuclear mass corrections are not included in Eq. (2) and are generally added as a firstorder perturbation correction after self-consistency is obtained. The transverse Breit interaction

$$
B_{i j}=-\frac{1}{2 r_{i j}}\left[\alpha_{i} \alpha_{j}+\frac{\left(\alpha_{i} r_{i j}\right)\left(\alpha_{j} r_{i j}\right)}{r_{i j}^{2}}\right]
$$

was included in subsequent CI calculations [26].

\section{Generation of configuration expansions}

In this work, there may be several CSFs that are important components to the wave-function expansion, defined in terms of orbitals with principal quantum number $n \leq 3$. These CSFs define a multireference set (MR). The most important corrections to the wave function arise from single and double excitations (SD) from orbitals occupied in the MR set to unfilled subshells. Because $\mathrm{W}^{62+}$ is so highly ionized, the most important contributions are CSFs within the $n=3$ complex that interact with one or more members of the MR set. The basis consists of spin orbitals: $2 s, 2 p, 3 s, 3 p, 3 d, 4 s, 4 p, 4 d, 5 s, 5 p, 5 d, 5 f, 4 f, 6 s, 6 p, 6 d$, and $6 f$ for tungsten system. The double excitations are limited only to configurations such as: $\{3 s n l\},\{3 p n l\}$, and $\{3 d n l\}$. That is, that one electron has been excited from the outermost core shell to the next open shell. We believe that these configurations are the most appropriate to represent core-valence correlations. Further corrections are included and the expansions increase in size significantly. The same radial functions are used in the wave-function expansion for all levels of the group in this extended optimal level (EOL) calculation.

In the last step, a relativistic configuration interaction (RCI) calculation was performed to include the Breit interaction as a first order perturbation corrections evaluated with electron-electron interactions in the Coulomb gauge, QED and finite nuclear mass corrections. Because the decrease in energy in going from $n=3$ to $n=5$ calculations was found to be fairly small and contributions from $n=6$ were expected to be even smaller, the rather large $n=6$ expansions for odd parity were condensed to eliminate CSFs with an expansion coefficient less than 0.0001 from the Dirac-Coulomb (DC) wavefunctions. Other variations of the correction amount is small. The effect of triple and quadruple excitations (TQ) from the reference set were neglected in this study.

\section{Results and discussions}

The MCDHF calculations were performed with the wave-function expanded in a basis of CSFs defined in terms of orbitals that are coupled in $j j$ coupling, and the results are presented in $L S J$ notation. If we make the same of the $n l_{-}$and $n l$ MCDHF radial functions, it is possible to transform the wave function from the jj coupling scheme to $L S J$ coupling. In Table I, we list the energy levels and the corresponding configurations that we consider in this work. Spectroscopic notations 
are used for these states, though this is no more than a convenience in labeling the energy levels in our present intermediate coupling calculations. Specifically, the sixteen states that we have studied. For simplicity, the symbols $p_{+}, p_{-}, d_{+}$, and $d_{-}$are used to denote the $p_{3 / 2}$, $p_{1 / 2}, d_{5 / 2}$, and $d_{3 / 2}$ orbitals, respectively, in Table I and throughout this work, while $p$ and $d$ simply refer to the nonrelativistic designations of the orbitals with $l=1,2$, respectively.

TABLE I

Possible two electron configurations in the $n=3$ complex, together with spectroscopic designations of the related eigenstates.

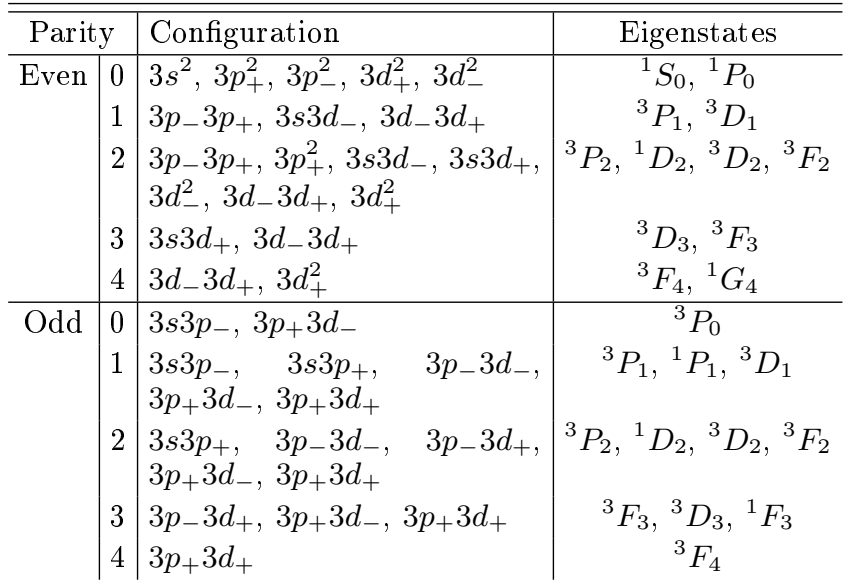

Table II summarizes the calculations performed for the twenty groups of levels by showing the $J$ values and parity of each group, the levels that were included in the optimization process, and the size of the wave-function expansion for the $n=6$ results reported as the final results for this paper. Contributions from higher partial waves with $l>3$ are small and are obtained by extrapolations.

\section{TABLE II}

Summary of the extended optimal level MCDHF calculations performed indicating the range of eigenvalues and the size of the interaction matrix for each $J$ of the group. The Mg-like lines have a $1 s^{2}, 2 s^{2} 2 p^{6} J^{\pi}=0^{+}$Ne-like core. Configuration state identifications are given by core + the respective valence configurations.

\begin{tabular}{c|c|c|c|c}
\hline \hline States & $J$ & Parity & Eigenvalues & Size \\
\hline $3 s^{2}$ & 0 & + & 1 & 1240 \\
$3 p^{2}$ & $0,1,2$ & + & $1-2,1,1-2$ & $9214,24181,32276$ \\
$3 s 3 d$ & $1,2,3$ & + & $1,1-2,1$ & $27093,36848,37730$ \\
$3 d^{2}$ & $0,1,2$ & + & $1-2,1,1-3$ & $22630,61475,86470$ \\
& 3,4 & + & $1,1-2$ & 92488,83214 \\
$3 s 3 p$ & $0,1,2$ & - & $1,1-2,1$ & $8002,21369,28056$ \\
$3 p 3 d$ & $0,1,2$ & - & $1,1-3,1-4$ & $32141,87845,121251$ \\
& 3,4 & - & $1-3,1$ & 127490,111067
\end{tabular}

In Table III, contributions to the transition energies arising from different parts of the Hamiltonian for several levels are shown. They include Dirac-Coulomb (DC)
TABLE III

Contributions from Dirac-Coulomb (DC), transverse photon (TP), and QED to the $n=3-3$ excitation energies $\left(10^{4} \mathrm{~cm}^{-1}\right)$ for tungsten ions.

\begin{tabular}{c|c|c|c|c|c}
\hline \hline \multicolumn{2}{c|}{ Lines } & DC & Breit (TP) & QED & Total \\
\hline $3 s 2$ & ${ }^{1} S_{0}$ & 0.000 & 0.000 & 0.000 & 0.000 \\
$3 s 3 p$ & ${ }^{3} P_{0}$ & 112.829 & 3.498 & -3.940 & 112.387 \\
& ${ }^{3} P_{1}$ & 125.887 & 3.266 & -4.010 & 125.143 \\
& ${ }^{1} P_{1}$ & 443.923 & 0.054 & -3.698 & 440.279 \\
& ${ }^{3} P_{2}$ & 413.760 & -0.137 & -3.519 & 410.104 \\
& ${ }^{3} P_{0}$ & 269.452 & 3.037 & -3.857 & 268.632 \\
& ${ }^{1} D_{2}$ & 556.112 & 1.124 & -3.680 & 553.556 \\
& ${ }^{3} P_{1}$ & 558.841 & -0.558 & -3.638 & 554.645 \\
& ${ }^{3} P_{2}$ & 862.619 & -0.739 & -3.357 & 858.523 \\
& ${ }^{1} S_{0}$ & 878.664 & -1.295 & -3.336 & 874.033 \\
& ${ }^{3} D_{1}$ & 586.293 & -1.077 & -2.253 & 582.963 \\
& ${ }^{3} D_{2}$ & 597.090 & -0.477 & -2.760 & 593.853 \\
& ${ }^{3} D_{3}$ & 653.608 & -3.234 & -0.122 & 650.252 \\
& ${ }^{1} D_{2}$ & 667.918 & -3.235 & -0.379 & 664.304 \\
& ${ }^{3} F_{2}$ & 705.004 & 4.838 & -5.779 & 704.063 \\
& ${ }^{3} D_{1}$ & 732.861 & 5.150 & -5.682 & 732.329 \\
& ${ }^{3} P_{2}$ & 788.822 & 3.458 & -5.736 & 786.544 \\
& ${ }^{3} F_{3}$ & 792.045 & 3.159 & -6.041 & 789.163 \\
& ${ }^{3} D_{2}$ & 1014.547 & 2.209 & -4.380 & 1012.376 \\
& ${ }^{3} P_{0}$ & 1022.092 & 2.673 & -4.188 & 1020.577 \\
& ${ }^{1} F_{3}$ & 1022.571 & 2.453 & -4.192 & 1020.832 \\
& ${ }^{3} P_{1}$ & 1022.648 & 2.129 & -3.848 & 1020.929 \\
& ${ }^{3} F_{4}$ & 1072.069 & 2.059 & -3.911 & 1070.217 \\
& ${ }^{1} D_{2}$ & 1081.551 & 1.925 & -3.715 & 1079.761 \\
& ${ }^{3} D_{3}$ & 1096.808 & 1.623 & -3.229 & 1095.202 \\
& ${ }^{1} P_{1}$ & 1106.625 & 1.519 & -2.925 & 1105.219 \\
& ${ }^{3} F_{2}$ & 1177.021 & 0.390 & -0.771 & 1176.640 \\
& ${ }^{3} P_{0}$ & 1197.424 & -1.651 & -1.585 & 1197.358 \\
& ${ }^{3} F_{3}$ & 1242.172 & -1.729 & 0.058 & 1240.501 \\
& ${ }^{1} S_{0}$ & 1343.165 & -1.114 & 0.086 & 1342.137 \\
& ${ }^{1} P_{2}$ & 1251.558 & 1.571 & -3.083 & 1250.046 \\
& ${ }^{1} G_{4}$ & 1254.980 & -2.479 & 0.060 & 1252.561 \\
& ${ }^{3} P_{1}$ & 1255.047 & -0.806 & -0.058 & 1254.299 \\
${ }^{3}$ & 1315.354 & -3.784 & 0.188 & 1311.758 \\
& 1322.441 & -3.102 & 0.190 & 1319.529 \\
& & & & \\
\hline
\end{tabular}

and frequency-dependent Breit energies from the present MCDHF calculations, along with the mass polarization and QED corrections, which are also calculated here. Transition energies are dominated by the Coulomb energies, with large corrections from the Breit and QED corrections for these high- $Z$ ions. While the Breit energies depend on the details of the electronic configurations and vary in magnitude from 540 to $51500 \mathrm{~cm}^{-1}$, most of the QED corrections are about $-40000 \mathrm{~cm}^{-1}$ in size as they are dominated by the QED energy of the $n=3-3$ transitions. We note that to keep the sizes of the CI expansions computationally manageable, most of the MCDHF results only included valence-valence (VV) and core-valence $(\mathrm{CV})$ correlations from single and double 
excitations from the reference states, and some with VV correlations only.For the Mg-like tungsten ions, the core is again the Ne-like ground state. With $3 l 3 l^{\prime}=3 s^{2} 3 p^{2}$, $3 s 3 d$ and $3 s 3 p, 3 p 3 d$ for the lower and upper states, respectively, the CI expansions include VV correlations $\left(2 s^{2} 2 p^{6} n l n^{\prime} l^{\prime}\right.$ and $\mathrm{CV}$ correlations from the $n=2$ core and $n=3$ valence shells $\left(2 s^{2} 2 p^{5} 3 \ln l n^{\prime} l^{\prime}, 2 s 2 p^{6} 3 \ln n^{\prime} l^{\prime}\right)$ with $n, n^{\prime} \leq 6$ and $l, l^{\prime} \leq 3$. In general, the accuracies of our RCI energies are limited by the extensiveness of the CI expansions, and theoretical uncertainties are assigned to the DC energies in Table III accordingly. The accuracies of the QED energies, on the other hand, are not as sensitive to correlation effects, and their uncertainties are estimates based on the approximated treatment of screening and relaxation corrections.

\section{TABLE IV}

Energy levels $\left(10^{4} \mathrm{~cm}^{-1}\right)$ of Mg-like W for the $n=3$ complex calculated using MCDHF and compared with other theoretical results. Percentage differences by comparing our results with those obtained using RMBPT code in Ref. [9] were given, according to the formula abs (EMCDHF - ERMBPT) $\times$ $100 / \max \{$ EMCDHF, ERMBPT $\}$.

\begin{tabular}{|c|c|c|c|c|c|c|c|}
\hline \multicolumn{2}{|c|}{ Lines } & \multirow{2}{*}{$\begin{array}{c}\text { MCDHF } \\
0.000\end{array}$} & \multirow{2}{*}{$\frac{\mathrm{RMBPT}^{a}}{0.000}$} & \multirow{2}{*}{$\frac{\text { COWAN }^{b}}{0.000}$} & \multirow{2}{*}{$\begin{array}{c}\text { HULLAC }^{c} \\
0.000\end{array}$} & \multirow{2}{*}{$\begin{array}{c}\begin{array}{c}\text { Auto- } \\
\text { structure }^{d}\end{array} \\
0.000\end{array}$} & \multirow{2}{*}{$\begin{array}{r}\text { Diff. [\%] } \\
0.000\end{array}$} \\
\hline $3 s 2$ & ${ }^{1} S_{0}$ & & & & & & \\
\hline \multirow[t]{4}{*}{$3 s 3 p$} & ${ }^{3} P_{0}$ & 112.387 & 112.645 & 114.982 & 113.608 & 112.562 & 0.229 \\
\hline & ${ }^{3} P_{1}$ & 125.143 & 125.215 & 127.152 & 126.758 & 125.790 & 0.057 \\
\hline & ${ }^{1} P_{1}$ & 440.279 & 440.293 & 439.974 & 441.470 & 440.251 & 0.003 \\
\hline & ${ }^{3} P_{2}$ & 410.104 & 410.409 & 412.536 & 410.945 & 409.900 & 0.074 \\
\hline \multirow[t]{5}{*}{$3 p 2$} & ${ }^{3} P_{0}$ & 268.632 & 268.759 & 270.683 & 272.158 & 274.968 & 0.047 \\
\hline & ${ }^{1} D_{2}$ & 553.556 & 553.530 & 558.368 & 555.712 & 556.549 & 0.005 \\
\hline & ${ }^{3} P_{1}$ & 554.645 & 555.145 & 558.509 & 557.268 & 558.282 & 0.090 \\
\hline & ${ }^{3} P_{2}$ & 858.523 & 858.787 & 860.716 & 860.827 & 859.462 & 0.031 \\
\hline & ${ }^{1} S_{0}$ & 874.033 & 874.208 & 874.388 & 876.010 & 874.724 & 0.020 \\
\hline \multirow[t]{4}{*}{$3 s 3 d$} & ${ }^{3} D_{1}$ & 582.963 & 582.670 & 577.298 & 584.271 & 583.247 & 0.050 \\
\hline & ${ }^{3} D_{2}$ & 593.853 & 593.043 & 586.261 & 595.431 & 594.454 & 0.130 \\
\hline & ${ }^{3} D_{3}$ & 650.252 & 649.786 & 645.147 & 651.468 & 652.835 & 0.072 \\
\hline & ${ }^{1} D_{2}$ & 664.304 & 663.829 & 655.165 & 665.679 & 667.456 & 0.072 \\
\hline \multirow[t]{12}{*}{$3 p 3 d$} & ${ }^{3} F_{2}$ & 704.063 & 704.092 & 703.292 & 707.300 & 714.879 & 0.004 \\
\hline & ${ }^{3} D_{1}$ & 732.329 & 731.902 & 726.852 & 735.578 & 742.252 & 0.058 \\
\hline & ${ }^{3} P_{2}$ & 786.544 & 786.474 & 783.297 & 789.170 & 798.440 & 0.009 \\
\hline & ${ }^{3} F_{3}$ & 789.163 & 788.874 & 786.096 & 792.345 & 801.438 & 0.037 \\
\hline & ${ }^{3} D_{2}$ & 1012.376 & 1012.367 & 1008.971 & 1014.869 & 1014.898 & 0.001 \\
\hline & ${ }^{3} P_{0}$ & 1020.577 & 1020.477 & 1015.569 & 1022.539 & 1022.501 & 0.010 \\
\hline & ${ }^{1} F_{3}$ & 1020.832 & 1020.553 & 1016.849 & 1023.518 & 1023.986 & 0.027 \\
\hline & ${ }^{3} P_{1}$ & 1020.929 & 1020.898 & 1017.192 & 1023.931 & 1023.322 & 0.003 \\
\hline & ${ }^{3} F_{4}$ & 1070.217 & 1070.330 & 1067.532 & 1072.554 & 1074.584 & 0.011 \\
\hline & ${ }^{1} D_{2}$ & 1079.761 & 1079.848 & 1076.372 & 1081.940 & 1083.717 & 0.008 \\
\hline & ${ }^{3} D_{3}$ & 1095.202 & 1094.835 & 1091.315 & 1097.831 & 1099.926 & 0.034 \\
\hline & ${ }^{1} P_{1}$ & 1105.219 & 1105.043 & 1100.087 & 1107.638 & 1109.845 & 0.016 \\
\hline \multirow[t]{9}{*}{$3 d 2$} & ${ }^{3} F_{2}$ & 1176.640 & 1176.471 & 1165.564 & 1180.174 & 1229.952 & 0.014 \\
\hline & ${ }^{3} P_{0}$ & 1197.358 & 1197.969 & 1183.042 & 1201.270 & 1250.128 & 0.051 \\
\hline & ${ }^{3} F_{3}$ & 1240.501 & 1240.320 & 1230.178 & 1243.219 & 1296.940 & 0.015 \\
\hline & ${ }^{3} P_{2}$ & 1250.046 & 1250.128 & 1238.383 & 1253.129 & 1305.991 & 0.007 \\
\hline & ${ }^{1} G_{4}$ & 1252.561 & 1251.834 & 1241.138 & 1256.067 & 1309.894 & 0.058 \\
\hline & ${ }^{3} P_{1}$ & 1254.299 & 1254.505 & 1241.300 & 1256.707 & 1309.806 & 0.016 \\
\hline & ${ }^{3} F_{4}$ & 1311.758 & 1311.439 & 1301.281 & 1314.624 & 1371.588 & 0.024 \\
\hline & ${ }^{1} D_{2}$ & 1319.529 & 1319.870 & 1307.795 & 1322.420 & 1378.769 & 0.026 \\
\hline & ${ }^{1} S_{0}$ & 1342.137 & 1343.117 & 1326.537 & 1345.248 & 1401.564 & 0.073 \\
\hline
\end{tabular}

${ }^{a}$ RMBPT code in Refs. [8, 9], ${ }^{b}$ COWAN code and ${ }^{c}$ HULLAC code in Ref. [8],

${ }^{d}$ Autostructure code in Ref [23]. 
The excitation energies for $\mathrm{W}^{62+}$ including MCDHF results have been reported and compared with other works available in Table IV. It is seen that there is a good agreement between our MCDHF results and other theoretical results of the relativistic many-body perturbation theory method (RMBPT code) $[8,9]$, and the Hartree-Fock-relativistic method (COWAN code) [8], and the multiconfiguration relativistic Hebrew University Lawrence Atomic Code (HULLAC code) [8], and the non-relativistic or semi-relativistic wave-functions of the Autostructure code [23]. However, a more detailed comparison of the calculated and other theoretical energies for these transitions indicates that the excitation energies given by our MCDHF calculations are in better agreement with the RMBPT energies. Hence we have given the formula abs $\left[E_{\mathrm{MCDHF}}-E_{\mathrm{RMBPT}}\right] \times$ $100 / \max \left\{E_{\mathrm{MCDHF}}, E_{\mathrm{RMBPT}}\right\}$, the differences in percent, for the accuracy of our results in the last columns of Table IV. When the difference (\%) between our resuts and RMBPT results is investigated, the differences are generally in the range of $0.00-0.229$ for all the results.

TABLE V

Wavelengths ( $\lambda$ in $\AA$ ), transition probabilities $\left(\mathrm{A} \mathrm{in} \mathrm{s}^{-1}\right)$ and line strengths $(S$ in a.u. $)$, to the $n=3-3$ transitions in Mg-like tungsten. Comparison with theoretical measurements from [8,9]. Column ninth gives the ratios of dipole length and velocity rates. The notation $\mathrm{a}(\mathrm{b})$ abbreviates $\mathrm{a} \times 10^{\mathrm{b}}$.

\begin{tabular}{|c|c|c|c|c|c|c|c|c|c|c|}
\hline \multicolumn{2}{|c|}{ Transition } & $\lambda_{\mathrm{MCDHF}}$ & $\lambda_{\mathrm{RMBPT}}^{b}$ & $\lambda_{\text {HULLAC }}^{c}$ & $A_{\mathrm{MCDHF}}$ & $A_{\mathrm{RMBPT}}^{b}$ & $A_{\text {HULLAC }}^{c}$ & $S_{\mathrm{MCDHF}}$ & $S_{\mathrm{RMBPT}}^{b}$ & $A_{\mathrm{L}} / A_{\mathrm{V}}$ \\
\hline $3 s 2{ }^{1} S_{0}$ & $3 s 3 p^{3} P_{1}$ & 79.909 & $79.860^{a}$ & 78.890 & $1.81(10)$ & $1.80(10)^{a}$ & $2.67(10)$ & $1.37(-2)$ & & 0.973 \\
\hline $3 s 2{ }^{1} S_{0}$ & $3 s 3 p{ }^{1} P_{1}$ & 22.713 & 22.712 & 22.650 & $2.57(12)$ & $2.57(12)$ & $3.28(12)$ & $4.46(-2)$ & $4.47(-2)$ & 0.989 \\
\hline $3 s 3 p{ }^{3} P_{1}$ & $3 s 3 d^{1} D_{2}$ & 18.520 & 18.566 & & $2.25(11)$ & $2.15(11)$ & & $3.52(-3)$ & $3.40(-3)$ & 1.017 \\
\hline $3 s 3 p{ }^{3} P_{0}$ & $3 s 3 d^{3} D_{1}$ & 21.247 & 21.275 & 21.250 & $1.10(12)$ & $1.09(12)$ & $1.16(12)$ & $1.57(-2)$ & $1.56(-2)$ & 1.004 \\
\hline $3 s 3 p{ }^{3} P_{1}$ & $3 s 3 d^{3} D_{2}$ & 21.323 & 21.375 & 21.340 & $2.79(12)$ & $2.73(12)$ & $2.81(12)$ & $6.67(-2)$ & $6.59(-2)$ & 1.017 \\
\hline $3 s 3 p{ }^{3} P_{1}$ & $3 s 3 d^{3} D_{1}$ & 21.836 & 21.860 & 21.860 & $4.99(11)$ & $5.03(11)$ & $4.62(11)$ & $7.69(-3)$ & $7.79(-3)$ & 1.001 \\
\hline $3 s 3 p{ }^{1} P_{1}$ & $3 s 3 d{ }^{1} D_{2}$ & 44.511 & 44.735 & & $2.09(11)$ & $2.02(11)$ & & $4.54(-2)$ & $4.47(-2)$ & 1.029 \\
\hline $3 s 3 p{ }^{3} P_{2}$ & $3 s 3 d^{3} D_{3}$ & 41.637 & 41.775 & 41.580 & $2.51(11)$ & $2.48(11)$ & $2.52(11)$ & $6.27(-2)$ & $6.26(-2)$ & 1.037 \\
\hline $3 s 3 p^{3} P_{2}$ & $3 s 3 d{ }^{1} D_{2}$ & 39.237 & $39.460^{a}$ & 39.260 & $7.39(10)$ & $7.32(10)^{a}$ & $7.89(10)$ & $1.12(-2)$ & & 1.024 \\
\hline $3 s 3 p p^{3} P_{1}$ & $3 p 2{ }^{3} P_{0}$ & 69.707 & 69.665 & & $1.28(11)$ & $1.28(11)$ & & $2.15(-2)$ & $2.14(-2)$ & 0.995 \\
\hline $3 s 3 p^{3} P_{1}$ & $3 p 2{ }^{1} D_{2}$ & 23.267 & & 23.347 & $4.78(11)$ & $4.72(11)$ & & $1.49(-2)$ & $1.48(-2)$ & 0.952 \\
\hline $3 s 3 p^{3} P_{2}$ & $3 p 2{ }^{1} D_{2}$ & 69.137 & $69.870^{a}$ & 69.070 & $3.26(10)$ & $3.17(10)^{a}$ & $3.61(10)$ & $2.66(-2)$ & & 0.973 \\
\hline $3 s 3 p^{3} P_{0}$ & $3 p 2{ }^{3} P_{1}$ & 22.605 & 22.599 & 22.540 & $1.21(12)$ & $1.20(12)$ & $1.29(12)$ & $2.08(-2)$ & $2.06(-2)$ & 0.992 \\
\hline $3 s 3 p^{3} P_{1}$ & $3 p 2{ }^{3} P_{1}$ & 23.272 & 23.260 & 23.230 & $6.39(11)$ & $6.50(11)$ & $5.90(11)$ & $1.19(-2)$ & $1.21(-2)$ & 0.991 \\
\hline $3 s 3 p{ }^{3} P_{2}$ & $3 p 2{ }^{3} P_{2}$ & 22.298 & 22.303 & & $1.82(12)$ & $1.82(12)$ & & $4.99(-2)$ & $4.99(-2)$ & 0.989 \\
\hline $3 s 3 p{ }^{1} P_{1}$ & $3 p 2{ }^{3} P_{2}$ & 5 & 23. & & $1.85(12)$ & $1.84(12)$ & & $6.24(-2)$ & $6.20(-2)$ & 0.995 \\
\hline $3 s 3 p{ }^{1} P_{1}$ & $3 p 2{ }^{1} S_{0}$ & 23.050 & 23.046 & & $3.58(12)$ & $3.57(12)$ & & $2.16(-2)$ & $2.16(-2)$ & 1.014 \\
\hline $3 s 3 d^{3} D_{1}$ & $3 p 3 d^{3} P_{1}$ & 23 & 22.819 & & $1.49(12)$ & $1.49(12)$ & & $2.62(-2)$ & $2.62(-2)$ & 1.049 \\
\hline $3 s 3 d^{3} D_{2}$ & $3 p 3 d^{3} D_{1}$ & 23.429 & 23.372 & & $5.25(11)$ & $5.22(11)$ & & $1.02(-2)$ & $9.88(-3)$ & 1.011 \\
\hline $3 s 3 d^{3} D_{1}$ & $3 p 3 d^{3} P_{2}$ & 23.283 & 23.272 & & $8.81(11)$ & $8.76(11)$ & & $2.77(-2)$ & $2.73(-2)$ & 0.991 \\
\hline $3 s 3 d^{3} D_{2}$ & $3 p 3 d^{3} P_{2}$ & 23.945 & 23.848 & & $2.46(11)$ & $2.57(11)$ & & $7.99(-3)$ & $8.62(-3)$ & 1.078 \\
\hline $3 s 3 d^{3} D_{3}$ & $3 p 3 d^{3} F_{3}$ & 71.969 & $71.900^{a}$ & 70.980 & $2.53(10)$ & $2.52(10)^{a}$ & $2.62(10)$ & $3.26(-2)$ & & 0.984 \\
\hline $3 s 3 d^{3} D_{3}$ & $3 p 3 d d^{3} D_{2}$ & 23.274 & 23.253 & 23.230 & $4.37(11)$ & $4.31(11)$ & $4.39(11)$ & $1.36(-2)$ & $1.34(-2)$ & 1.014 \\
\hline $3 s 3 d^{1} D_{2}$ & $3 p 3 d{ }^{3} D_{2}$ & 24.096 & 24.037 & & $1.35(12)$ & $1.35(12)$ & & $4.65(-2)$ & $4.64(-2)$ & 1.046 \\
\hline $3 s 3 d^{3} D_{1}$ & $3 p 3 d^{3} P_{0}$ & 22.847 & 22.841 & & $2.01(12)$ & $1.98(12)$ & & $1.18(-2)$ & $1.16(-2)$ & 1.047 \\
\hline $3 s 3 d^{3} D_{3}$ & $3 p 3 d^{1} F_{3}$ & 22.467 & 22.469 & & $9.97(11)$ & $9.90(11)$ & & $3.90(-2)$ & $3.88(-2)$ & 1.005 \\
\hline $3 s 3 d^{1} D_{2}$ & $3 p 3 d^{1} F_{3}$ & 23.232 & 23.202 & & $6.72(11)$ & $6.50(11)$ & & $2.91(-2)$ & $2.81(-2)$ & 0.993 \\
\hline $3 s 3 d^{3} D_{3}$ & $3 p 3 d^{3} F_{4}$ & 23.802 & 23.779 & 23.750 & $1.63(12)$ & $1.62(12)$ & $1.65(12)$ & $9.72(-2)$ & $9.67(-2)$ & 0.976 \\
\hline $3 s 3 d^{3} D_{2}$ & $3 p 3 d^{3} D_{3}$ & 23.465 & 23.391 & & $2.66(12)$ & $2.59(12)$ & & $1.19(-1)$ & $1.14(-1)$ & 0.967 \\
\hline $3 s 3 d^{1} D_{2}$ & $3 p 3 d^{1} P_{1}$ & 22.680 & & & $1.88(12)$ & $1.85(12)$ & & $3.26(-2)$ & $3.20(-2)$ & 1.056 \\
\hline $3 s 3 d^{3} D_{3}$ & $3 p 3 d^{3} P_{2}$ & 73.350 & $73.160^{a}$ & 72.620 & $4.22(10)$ & $4.20(10)^{a}$ & $4.33(10)$ & $4.11(-2)$ & & 1.022 \\
\hline $3 p 2{ }^{3} P_{1}$ & $3 p 3 d^{3} D_{1}$ & 56.279 & $56.570^{a}$ & 56.080 & $1.59(10)$ & $1.59(10)^{a}$ & $1.57(10)$ & $4.20(-3)$ & & 0.975 \\
\hline $3 p 2{ }^{1} D_{2}$ & $3 p 3 d^{3} P_{2}$ & 21.833 & 21.794 & 21.780 & $1.30(12)$ & $1.45(12)$ & $1.55(12)$ & $3.34(-2)$ & $3.71(-2)$ & 1.000 \\
\hline $3 p 2{ }^{3} P_{1}$ & $3 p 3 d^{3} P_{2}$ & 21.846 & 21.871 & 21.850 & $7.37(11)$ & $7.20(11)$ & $7.85(11)$ & $1.89(-2)$ & $1.86(-2)$ & 1.003 \\
\hline $3 p 2{ }^{1} D_{2}$ & $3 p 3 d^{3} F_{3}$ & 42.509 & 42.491 & & $1.43(11)$ & $1.45(11)$ & & $3.81(-3)$ & $3.85(-2)$ & 1.014 \\
\hline $3 p 2{ }^{3} P_{2}$ & $3 p 3 d^{3} D_{2}$ & 45.178 & 45.236 & & $1.22(11)$ & $1.23(11)$ & & $2.78(-2)$ & $2.81(-2)$ & 1.051 \\
\hline $3 p 2{ }^{3} P_{2}$ & $3 p 3 d{ }^{1} F_{3}$ & 42.232 & 42.364 & & $4.49(11)$ & $4.41(11)$ & & $1.17(-1)$ & $1.16(-1)$ & 1.002 \\
\hline $3 p 2{ }^{3} P_{1}$ & $3 p 3 d^{3} P_{0}$ & 21.462 & 21.490 & & $1.50(12)$ & $1.47(12)$ & & $7.33(-3)$ & $7.20(-3)$ & 1.034 \\
\hline
\end{tabular}


TABLE V cont.

\begin{tabular}{|c|c|c|c|c|c|c|c|c|c|c|}
\hline \multicolumn{2}{|c|}{ Transition } & $\lambda_{\mathrm{MCDHF}}$ & $\lambda_{\mathrm{RMBPT}}^{b}$ & $\lambda_{\text {HULLAC }}^{c}$ & $A_{\mathrm{MCDHF}}$ & $A_{\mathrm{RMBPT}}^{b}$ & $A_{\text {HULLAC }}^{c}$ & $S_{\mathrm{MCDHF}}$ & $S_{\mathrm{RMBPT}}^{b}$ & $A_{\mathrm{L}} / A_{\mathrm{V}}$ \\
\hline $3 p 2{ }^{3} P_{1}$ & $3 p 3 d^{3} P_{1}$ & 21.441 & $21.470^{a}$ & 21.450 & $1.31(12)$ & $1.32(12)^{a}$ & $1.34(12)$ & $1.91(-2)$ & & 1.015 \\
\hline $3 p 2{ }^{3} P_{1}$ & $3 p 3 d^{1} D_{2}$ & 43.122 & 43.228 & & $2.10(11)$ & $2.08(11)$ & & $4.17(-2)$ & $4.16(-2)$ & 1.050 \\
\hline $3 p 2{ }^{3} P_{0}$ & $3 p 3 d^{3} P_{1}$ & 21.558 & 21.592 & & $2.09(12)$ & $2.08(12)$ & & $3.10(-2)$ & $3.10(-2)$ & 1.015 \\
\hline $3 p 2{ }^{1} S_{0}$ & $3 p 3 d^{1} P_{1}$ & 43.147 & 43.321 & & $2.38(11)$ & $2.35(11)$ & & $2.84(-3)$ & $2.83(-2)$ & 1.071 \\
\hline $3 p 2{ }^{1} D_{2}$ & $3 p 3 d^{3} D_{3}$ & 21.432 & 21.412 & 21.360 & $3.68(11)$ & $3.58(11)$ & $3.79(11)$ & $1.27(-2)$ & $1.21(-2)$ & 1.042 \\
\hline $3 p 3 d{ }^{1} D_{2}$ & $3 d 2{ }^{3} P_{1}$ & 21.373 & 21.366 & & $1.63(12)$ & $1.65(12)$ & & $2.36(-2)$ & $2.38(-2)$ & 0.951 \\
\hline $3 p 3 d{ }^{3} F_{2}$ & $3 d 2{ }^{3} F_{2}$ & 21.161 & 21.169 & & $1.61(12)$ & $1.62(12)$ & & $3.76(-2)$ & $3.78(-2)$ & 1.066 \\
\hline $3 p 3 d{ }^{3} P_{1}$ & $3 d 2{ }^{3} P_{0}$ & 21.496 & 21.456 & & $3.38(12)$ & $3.30(12)$ & & $1.66(-2)$ & $1.61(-2)$ & 0.899 \\
\hline $3 p 3 d{ }^{1} D_{2}$ & $3 d 2{ }^{3} P_{2}$ & 21.570 & 21.568 & & $1.22(12)$ & $1.23(12)$ & & $3.01(-2)$ & $3.05(-2)$ & 1.025 \\
\hline $3 p 3 d{ }^{3} F_{3}$ & $3 d 2{ }^{3} F_{4}$ & 21.577 & 21.600 & & $1.26(12)$ & $1.21(12)$ & & $5.65(-2)$ & $5.44(-2)$ & 0.934 \\
\hline $3 p 3 d{ }^{3} F_{3}$ & $3 d 2{ }^{3} P_{2}$ & 21.692 & 21.680 & & $3.70(11)$ & $3.72(11)$ & & $9.38(-3)$ & $9.36(-3)$ & 0.893 \\
\hline $3 p 3 d{ }^{1} D_{2}$ & $3 d 2{ }^{3} F_{3}$ & 22.026 & 22.034 & & $6.27(11)$ & $6.10(11)$ & & $2.31(-2)$ & $2.26(-2)$ & 0.986 \\
\hline $3 p 3 d{ }^{3} F_{3}$ & $3 d 2{ }^{3} F_{3}$ & 22.150 & 22.151 & & $8.90(11)$ & $8.84(11)$ & & $3.35(-2)$ & $3.32(-2)$ & 1.068 \\
\hline $3 p 3 d{ }^{3} P_{1}$ & $3 d 2{ }^{3} F_{2}$ & 22.495 & 22.494 & & $2.28(12)$ & $2.30(12)$ & & $3.94(-2)$ & $3.88(-2)$ & 0.972 \\
\hline $3 p 3 d^{3} F_{4}$ & $3 d 2{ }^{1} G_{4}$ & 41.410 & 41.475 & 41.31 & $1.19(11)$ & $1.18(11)$ & $1.19(11)$ & $3.75(-2)$ & $3.74(-2)$ & 1.140 \\
\hline $3 p 3 d^{3} D_{2}$ & $3 d 2{ }^{1} D_{2}$ & 41.708 & 41.663 & & $3.10(11)$ & $3.26(11)$ & & $5.53(-2)$ & $5.83(-2)$ & 0.993 \\
\hline $3 p 3 d{ }^{1} P_{1}$ & $3 d 2{ }^{1} S_{0}$ & 42.401 & 42.004 & & $6.31(11)$ & $6.03(11)$ & & $2.37(-2)$ & $2.21(-2)$ & 0.930 \\
\hline $3 p 3 d^{3} D_{1}$ & $3 d 2{ }^{3} P_{1}$ & 42.854 & 42.807 & & $1.14(11)$ & $1.20(11)$ & & $1.32(-2)$ & $1.40(-2)$ & 0.927 \\
\hline $3 p 3 d^{3} D_{3}$ & $3 d 2{ }^{3} F_{4}$ & 43.174 & 43.237 & & $2.59(11)$ & $2.52(11)$ & & $9.26(-2)$ & $9.04(-2)$ & 0.899 \\
\hline $3 p 3 d^{3} D_{1}$ & $3 d 2{ }^{3} P_{2}$ & 43.626 & 43.624 & & $2.11(11)$ & $2.21(11)$ & & $2.69(-2)$ & $2.71(-2)$ & 0.949 \\
\hline $3 p 3 d{ }^{3} P_{2}$ & $3 d 2{ }^{3} F_{3}$ & 43.848 & 43.869 & & $1.77(11)$ & $1.74(11)$ & & $5.17(-2)$ & $5.07(-2)$ & 0.984 \\
\hline $3 p 3 d{ }^{1} F_{3}$ & $3 d 2{ }^{1} G_{4}$ & 46.167 & 46.167 & & $3.05(11)$ & $2.98(11)$ & & $1.33(-1)$ & $1.30(-1)$ & 0.916 \\
\hline $3 p 3 d{ }^{1} P_{1}$ & $3 d 2{ }^{1} D_{2}$ & 46.659 & 46.549 & & $1.76(11)$ & $1.79(11)$ & & $2.66(-2)$ & $2.68(-2)$ & 0.898 \\
\hline $3 p 3 d^{3} D_{3}$ & $3 d 2{ }^{3} P_{2}$ & 64.562 & $64.390^{a}$ & 64.390 & $3.92(9)$ & $4.16(9)^{a}$ & $3.42(9)$ & $2.72(-3)$ & & 0.972 \\
\hline $3 p 3 d^{3} F_{4}$ & $3 d 2{ }^{3} F_{3}$ & 58.745 & $58.830^{a}$ & 58.590 & $4.91(9)$ & $4.88(9)^{a}$ & $4.88(9)$ & $3.44(-3)$ & & 0.984 \\
\hline
\end{tabular}

${ }^{a}$ Safronova et al. [8], ${ }^{b}$ RMBPT code in Ref. [9], ${ }^{c}$ HULLAC code in Ref [8].

In Table $\mathrm{V}$ we compare our results for wavelengths $\lambda$, transition probabilities $A$, and line strengths $S$, and the ratios of velocity to length rates $\left(A_{L} / A_{V}\right)$ in Mg-like tungsten. It is clear that the transition wavelengths are in general in very good agreement with the MBPT calculation of Safronova et al. [8, 9] and the HULLAC calculations of Safronova et al. [8], except for some transitions with a maximum difference of approximately $1.37 \%$. The calculations are fundamentally different and a comparison between the different models employed merely indicates the main similarities, and only the final converged values can be critically compared. To avoid future level identification problems, besides wavelengths we include in Table $\mathrm{V}$ transition rates $A$ and line strength $S$. Our GRASP2 K calculation of the $A$ and $S$ values for the $n=3-3$ transitions are in excellent agreement with the theoretical results $[8,9]$. In order to check our calculations, the ratios of velocity to length strengths $\left(A_{L} / A_{V}\right)$ were computed, and we find that most ratios fluctuate around 1 . The near equal values of the length and velocity rates for most of the transitions as seen from the last column of Table $\mathrm{V}$ provide one reasonable measure of the reliability of the present calculated results.

\section{TABLE VI}

Transition probabilities $\left(A\right.$ in $\left.\mathrm{s}^{-1}\right)$ for M1 and $\mathrm{E} 2$ transitions in Mg-like tungsten. Comparison with theoretical measurements from GRASPVU [7] and Autostructure [23]. The notation a(b) abbreviates a $\times 10^{\mathrm{b}}$.

\begin{tabular}{c|c|c|c|c|c|c|c}
\hline \hline \multirow{2}{*}{$\begin{array}{c}\text { Lower } \\
\text { level }\end{array}$} & \multirow{2}{*}{$\begin{array}{c}\text { Upper } \\
\text { level }\end{array}$} & \multicolumn{3}{|c|}{$A^{\mathrm{M} 1}\left[\mathrm{~s}^{-1}\right]$} & \multicolumn{3}{c}{$A^{\mathrm{E} 2}\left[\mathrm{~s}^{-1}\right]$} \\
\cline { 3 - 8 } & GRASP2K & GRASPVU & $\begin{array}{c}\text { Auto- } \\
\text { structure }\end{array}$ & GRASP2k & GRASPVU & $\begin{array}{c}\text { Auto- } \\
\text { structure }\end{array}$ \\
\hline $3 s 3 p{ }^{3} P_{0}$ & $3 s 3 p{ }^{3} P_{1}$ & $2.66(4)$ & $2.60(4)$ & $3.03(4)$ & & & \\
$3 s 3 p{ }^{3} P_{0}$ & $3 s 3 p{ }^{3} P_{2}$ & & & & $2.91(6)$ & $2.91(6)$ & $2.95(6)$ \\
$3 s 3 p{ }^{3} P_{1}$ & $3 s 3 p{ }^{3} P_{2}$ & $2.20(8)$ & $2.19(8)$ & $2.22(8)$ & $3.86(6)$ & $3.86(6)$ & $3.88(6)$ \\
$3 s 3 p{ }^{3} P_{0}$ & $3 s 3 p{ }^{1} P_{1}$ & $1.68(8)$ & $1.67(8)$ & $1.70(8)$ & & & \\
$3 s 3 p{ }^{3} P_{1}$ & $3 s 3 p{ }^{1} P_{1}$ & $8.14(7)$ & $8.13(7)$ & $8.21(7)$ & $7.08(6)$ & $7.07(6)$ & $7.15(6)$ \\
$3 s 3 p{ }^{3} P_{2}$ & $3 s 3 p{ }^{1} P_{1}$ & $1.67(5)$ & $1.63(5)$ & $1.71(5)$ & $3.63(1)$ & $3.50(1)$ & $3.71(1)$
\end{tabular}


In Table VI, we list transition rates $A$ for the 5 M1 and $4 \mathrm{E} 2$ transitions in $\mathrm{W}^{62+}$. There are a few data on E2, and M1 forbidden transitions for Mg-like tungsten in available literature. Therefore, we have only compared the results obtained with other theoretical results [7, 23]. Our GRASP2K transition rates for the $3 s 3 p-3 s 3 p$ transition in Mg-like tungsten are in better agreement with the GRASPVU data than the FAC results.

TABLE VII

Wavelengths ( $\lambda$ in $\AA$ ), transition probabilities $\left(A\right.$ in s$\left.{ }^{-1}\right)$ and line strengths ( $S$ in a.u.) of forbidden lines (E2, M1, and M2). The notation $\mathrm{a}(\mathrm{b})$ abbreviates $\mathrm{a} \times 10^{\mathrm{b}}$.

\begin{tabular}{|c|c|c|c|c|c|}
\hline \multicolumn{2}{|c|}{ Transitions } & \multirow{2}{*}{\begin{tabular}{|c|}
$\begin{array}{c}\text { Transition } \\
\text { type }\end{array}$ \\
M1
\end{tabular}} & \multirow{2}{*}{$\begin{array}{c}\lambda[\AA] \\
788.662\end{array}$} & \multirow{2}{*}{\begin{tabular}{|l}
$A\left[\mathrm{~s}^{-1}\right]$ \\
$2.66(4)$
\end{tabular}} & \multirow{2}{*}{$\frac{S \text { [a.u.] }}{1.93(-5)}$} \\
\hline $3 s 3 p^{3} P_{0}$ & $s 3 p^{3} P_{1}$ & & & & \\
\hline $3 s 3 p^{3} P_{0}$ & $3 s 3 p^{3} P_{2}$ & $\mathrm{E} 2$ & 33.575 & $2.91(6)$ & $2.72(-7)$ \\
\hline \multirow[t]{2}{*}{$3 s 3 p^{3} P_{1}$} & $3 s 3 p^{3} P_{2}$ & 22 & 35.067 & $3.86(6)$ & $4.11(-7)$ \\
\hline & & $\mathrm{M}$ & 35.067 & 2.20 & \\
\hline $3 s 3 p^{3} P_{0}$ & $3 s 3 p{ }^{1} P_{1}$ & M1 & 30.486 & $1.68(8)$ & $-6)$ \\
\hline \multirow[t]{2}{*}{$3 s 3 p{ }^{3} P_{2}$} & $3 s 3 p{ }^{1} P_{1}$ & $\mathrm{E} 2$ & 331.334 & $3.63(1)$ & $(-9)$ \\
\hline & & M & 331.334 & $1.67(5)$ & $-6)$ \\
\hline $3 s 2{ }^{1} S_{0}$ & $3 s 3 p^{3} P_{2}$ & M2 & 24.384 & 2.3 & $(-9)$ \\
\hline $3 p 2{ }^{3} P_{0}$ & $3 p 2{ }^{3} P_{2}$ & $\mathrm{E} 2$ & 34.923 & $3.58(6)$ & $(-7)$ \\
\hline \multirow[t]{2}{*}{$3 p 2{ }^{3} P_{1}$} & $3 p 2{ }^{1} D_{2}$ & $\mathrm{E} 2$ & 32.901 & $4.32(6)$ & $(-7)$ \\
\hline & & M & & & \\
\hline $3 p 2{ }^{3} P_{2}$ & $3 p 2{ }^{1} S_{0}$ & E2 & 31.314 & 7) & $(-7)$ \\
\hline $3 s 3 p^{3} P_{0}$ & $3 p 2{ }^{3} P_{2}$ & $\mathrm{M}$ & 99 & & \\
\hline $3 s 3 p^{3} P_{0}$ & $3 p 2{ }^{1} D_{2}$ & 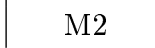 & 3.399 & & 6.2 \\
\hline $3 s 3 p^{3} P_{1}$ & $3 p 2{ }^{3} P_{1}$ & & 72 & & \\
\hline $3 s 3 p^{3} P_{1}$ & $3 p 2{ }^{3} P_{2}$ & 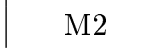 & 3.267 & 1.9 & $(-9)$ \\
\hline $3 s 3 p^{3} P_{1}$ & $3 p 2{ }^{1} D_{2}$ & & & & 6.3 \\
\hline $3 s 3 p{ }^{1} P_{1}$ & $3 p 2{ }^{1} D_{2}$ & M2 & 06 & & $(-9)$ \\
\hline $3 s 3 p^{3} P_{2}$ & & & & & \\
\hline $3 s 3 p^{3} P_{2}$ & $3 p 2{ }^{3} P_{1}$ & & 89 & 8.9 & 4.3 \\
\hline $3 s 3 p^{3} P_{2}$ & $3 p 2{ }^{3} P_{2}$ & & & & 1.0 \\
\hline $3 s 3 p^{3} P_{2}$ & $3 p 2{ }^{1} D_{2}$ & & 98 & & \\
\hline \multirow[t]{2}{*}{$3 s 3 d^{3} D_{1}$} & $3 s 3 d^{1} D_{2}$ & & & & \\
\hline & & & & & \\
\hline $3 s 3 d^{3} D_{1}$ & $3 s 3 d^{3} D_{3}$ & & 14 & & \\
\hline $3 s 3 p^{3} P_{0}$ & $3 s 3 d^{3} D_{2}$ & & 23 & & $9.01(-10)$ \\
\hline & & & & & 8. \\
\hline $3 s 3 p^{3} P_{1}$ & $3 s 3 d^{3} D_{1}$ & & 1.835 & $1.04(4)$ & $1.60(-10)$ \\
\hline $3 s 3 p{ }^{1} P_{1}$ & $3 s 3 d^{3} D_{1}$ & & 85 & $1.41(-1)$ & $7.17(-14)$ \\
\hline $3 s 3 p^{3} P_{1}$ & $3 s 3 d^{3} D_{2}$ & $\mathrm{~N}$ & 21.323 & $1.45(5)$ & $3.44(-9)$ \\
\hline $3 s 3 p^{3} P_{1}$ & $3 s 3 d{ }^{1} D_{2}$ & 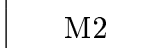 & 20 & $2.63(4)$ & $4.12(-10)$ \\
\hline $3 s 3 p{ }^{1} P_{1}$ & $3 s 3 d^{3} D_{2}$ & $\mathrm{~N}$ & 64.694 & $4.49(1)$ & $3.00(-11)$ \\
\hline $3 s 3 p{ }^{1} P_{1}$ & $3 s 3 d{ }^{1} D_{2}$ & 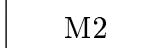 & 11 & $1.18(4)$ & $2.56(-9)$ \\
\hline $3 s 3 p^{3} P_{1}$ & $3 s 3 d^{3} D_{3}$ & $\mathrm{M}$ & 19.038 & $1.84(5)$ & $4.40(-9)$ \\
\hline $3 s 3 p{ }^{1} P_{1}$ & $3 s 3 d^{3} D_{3}$ & & 47.625 & $2.08(3)$ & $7.78(-10)$ \\
\hline $3 s 3 p^{3} P_{2}$ & $3 s 3 d^{3} D_{1}$ & M2 & 57.843 & $1.09(-1)$ & $3.12(-14)$ \\
\hline $3 s 3 p^{3} P_{2}$ & $3 s 3 d^{3} D_{2}$ & & 54.122 & $5.02(1)$ & $1.96(-11)$ \\
\hline $3 s 3 p^{3} P_{2}$ & $3 s 3 d{ }^{1} D_{2}$ & M2 & 39.237 & $1.12(4)$ & $1.67(-9)$ \\
\hline $3 s 3 p^{3} P_{2}$ & $3 s 3 d^{3} D_{3}$ & M2 & 41.637 & $1.84(4)$ & $4.60(-9)$ \\
\hline \multirow[t]{2}{*}{$3 p 3 d^{3} D_{1}$} & $3 p 3 d^{3} D_{2}$ & $\mathrm{E}$ & 184.340 & $1.50(2)$ & $2.15(-9)$ \\
\hline & & M1 & 184.340 & $1.57(6)$ & $2.42(-5)$ \\
\hline
\end{tabular}

TABLE VII cont.

\begin{tabular}{|c|c|c|c|c|c|}
\hline \multicolumn{2}{|c|}{ Transitions } & ransition & $\lambda[\AA]$ & $A\left[\mathrm{~s}^{-1}\right]$ & $S$ [a.u.] \\
\hline \multirow[t]{2}{*}{$3 p 3 d^{3} D_{1}$} & $p 3 d^{3}$ & $\mathrm{E} 2$ & 35.704 & $1.29(6)$ & $1.45(-7)$ \\
\hline & & 1011 & 35.704 & $1.07(8)$ & $(-5)$ \\
\hline \multirow[t]{2}{*}{$3 p 3 d^{3} D_{1}$} & $3 p 3 d{ }^{1} D_{2}$ & E2 & 28.770 & $2.36(5)$ & $1.32(-8)$ \\
\hline & & M1 & 8.770 & & \\
\hline \multirow[t]{2}{*}{$3 p 3 d^{3} F_{2}$} & $3 p 3 d d^{3} D_{3}$ & E2 & 31.559 & $3.23(6)$ & $-7)$ \\
\hline & & M1 & & & \\
\hline $3 p 3 d^{3} P_{1}$ & $3 p 3 d{ }^{1} F_{3}$ & $\mathrm{E} 2$ & 134.615 & $2.65(2)$ & $(-9)$ \\
\hline $3 p 3 d^{3} F_{2}$ & $3 p 3 d^{3} P_{0}$ & $\mathrm{E} 2$ & 1.594 & $9.37(6)$ & $(-7)$ \\
\hline \multirow[t]{2}{*}{$3 p 3 d^{3} F_{2}$} & $3 p 3 d d^{3} D_{1}$ & E2 & 354.061 & $1.30(1)$ & 8.5 \\
\hline & & M1 & & & \\
\hline \multirow[t]{2}{*}{$3 p 3 d^{3} F_{2}$} & $3 p 3 d$ & E2 & 31.549 & $8.16(6)$ & $(-7)$ \\
\hline & & M1 & & & \\
\hline \multirow[t]{2}{*}{$3 p 3 d^{3} F_{2}$} & $3 p 3$ & E2 & 4.892 & 1.7 & $-9)$ \\
\hline & & M1 & & & \\
\hline \multirow[t]{2}{*}{$3 p 3 d^{3} D_{2}$} & $3 p 3$ & E2 & 23 & & \\
\hline & & M1 & & & \\
\hline \multirow[t]{2}{*}{$3 p 3 d^{3} D_{2}$} & $3 p 3$ & $\mathrm{E} 2$ & 107.047 & & \\
\hline & & M1 & 10 & & \\
\hline \multirow[t]{2}{*}{$3 p 3 d{ }^{1} D_{2}$} & $3 p 3$ & $\mathrm{E} 2$ & & & \\
\hline & & M1 & & & \\
\hline \multirow[t]{2}{*}{$3 p 3 d^{3} F_{2}$} & $3 p 3$ & E2 & & & \\
\hline & & M1 & & & \\
\hline \multirow[t]{2}{*}{$3 p 3 d^{3} F_{2}$} & $3 p 3 d^{3} D_{3}$ & E2 & & & \\
\hline & & M1 & & & \\
\hline \multirow[t]{2}{*}{$3 p 3 d^{3} F_{2}$} & $3 p 3 d{ }^{1} F_{3}$ & 2 & & & \\
\hline & & M & & & \\
\hline \multirow[t]{2}{*}{$3 p 3 d^{3} D_{2}$} & $3 p 3 d{ }^{1} F_{3}$ & E2 & & & \\
\hline & & M1 & & & \\
\hline $3 p 3 d^{3} F_{2}$ & & E2 & & & 2.6 \\
\hline $3 p 3 d^{3} D_{2}$ & $3 p 3 d^{3} F_{4}$ & $\mathrm{E}$ & 37 & & \\
\hline \multirow[t]{2}{*}{$3 p 3 d^{3} F_{3}$} & $3 p 3 d{ }^{1} D_{2}$ & 2 & & & \\
\hline & & & & & \\
\hline \multirow[t]{2}{*}{$3 p 3 d^{3} D_{3}$} & $3 p 3 c$ & 2 & & & \\
\hline & & & & & \\
\hline \multirow[t]{2}{*}{$3 p 3 d d^{3} F_{2}$} & $3 p 3$ & $\mathrm{E}$ & & & \\
\hline & & & & & \\
\hline \multirow[t]{2}{*}{$3 d 2{ }^{3} F_{2}$} & $3 d 2$ & & 39 & & $-9)$ \\
\hline & & & & & \\
\hline $3 d 2{ }^{3} P_{2}$ & $3 d 2$ & 2 & & & $-9)$ \\
\hline \multirow[t]{2}{*}{$3 d 2{ }^{3} F_{3}$} & $3 d 2{ }^{1} D_{2}$ & $\mathrm{E}$ & 38 & & \\
\hline & & 1 & & & \\
\hline $3 d 2{ }^{3} F_{3}$ & $3 d 2{ }^{3} F_{4}$ & E2 & 829.197 & 3.7 & $9.50(-12)$ \\
\hline & & M1 & & & \\
\hline $3 d 2{ }^{3} F_{3}$ & $3 d 2{ }^{1} G_{4}$ & E2 & 140.337 & $1.02(2)$ & $1.25(-9)$ \\
\hline & & & & & $(-5)$ \\
\hline $3 s 3 d^{3} D_{1}$ & $3 p 3 d^{3} P_{1}$ & M2 & 22.823 & $3.87(4)$ & $6.81(-10)$ \\
\hline $3 s 3 d^{3} D_{2}$ & $3 p 3 d^{3} D_{1}$ & M2 & 23.429 & $2.84(5)$ & $5.43(-9)$ \\
\hline $3 s 3 d^{3} D_{1}$ & $3 p 3 d{ }^{3} P_{2}$ & M2 & 23.283 & $1.08(5)$ & $3.37(-9)$ \\
\hline $3 s 3 d^{3} D_{2}$ & $3 p 3 d^{3} P_{2}$ & M2 & 23.945 & $1.57(5)$ & $5.31(-9)$ \\
\hline $3 s 3 d^{3} D_{3}$ & $3 p 3 d^{3} F_{3}$ & M2 & 71.969 & $2.98(-1)$ & $3.83(-13)$ \\
\hline $3 s 3 d^{3} D_{3}$ & $3 p 3 d d^{3} D_{2}$ & M2 & 23.274 & $2.98(5)$ & $9.27(-9)$ \\
\hline $3 s 3 d^{1} D_{2}$ & $3 p 3 d^{3} D_{2}$ & M2 & 24.096 & $2.44(4)$ & $8.42(-10)$ \\
\hline $3 s 3 d^{1} D_{2}$ & $3 p 3 d^{3} P_{1}$ & M2 & 28.079 & $4.83(3)$ & $1.58(-10)$ \\
\hline
\end{tabular}


TABLE VII cont.

\begin{tabular}{|c|c|c|c|c|c|}
\hline \multicolumn{2}{|c|}{ Transitions } & Transition & $\lambda[\AA]$ & $A\left[\mathrm{~s}^{-1}\right]$ & $S$ [a.u.] \\
\hline$s 3 d^{3} D_{2}$ & $3 d^{1} F_{3}$ & M2 & 23.465 & $1.33(5)$ & U.9. (-9) \\
\hline $3 s 3 d^{3} D_{3}$ & $03 d^{1} F_{3}$ & 12 & 2.467 & $2.51(5)$ & $9.85(-9)$ \\
\hline $3 s 3 d{ }^{1} D_{2}$ & $p 3 d{ }^{1} F_{3}$ & 12 & 3.232 & $1.85(5)$ & $8.01(-9)$ \\
\hline $3 s 3 d^{3} D_{3}$ & $3 p 3 d^{3} F_{4}$ & 12 & 3.802 & $1.07(5)$ & $6.42(-9)$ \\
\hline $3 s 3 d^{3} D_{1}$ & $3 p 3 d d^{1} D_{2}$ & 12 & 20.121 & $1.17(3)$ & $2.34(-11)$ \\
\hline $3 s 3 d^{3} D_{2}$ & $3 p 3 d{ }^{1} D_{2}$ & 12 & 0.613 & $2.32(4)$ & $5.02(-10)$ \\
\hline $3 s 3 d^{3} D_{3}$ & $3 p 3 d{ }^{1} D_{2}$ & {$[2$} & 23.274 & $2.98(5)$ & $9.27(-9)$ \\
\hline $3 s 3 d^{3} D_{2}$ & $3 p 3 d^{3} D_{3}$ & {$[2$} & 3.465 & 1.3 & $(-9)$ \\
\hline $3 s 3 d^{3} D_{1}$ & $3 d{ }^{1} P_{1}$ & 2 & 24 & $5.70(1)$ & 5.91 \\
\hline $3 s 3 d{ }^{1} D_{2}$ & $3 p 3 d{ }^{1} P_{1}$ & 2 & 80 & 2.8 & $(-10)$ \\
\hline $3 s 3 d^{3} D_{3}$ & $3 p 3 d^{3} P_{2}$ & & & 2.9 & \\
\hline $3 p 2{ }^{3} P_{1}$ & $3 p 3 d^{3} D_{1}$ & 2 & 56.279 & $6.99(0)$ & $1.84(-12)$ \\
\hline $3 p 2{ }^{1} D_{2}$ & $3 p 3 d^{3} P_{2}$ & & 21.833 & 4) & $-9)$ \\
\hline $3 p 2{ }^{3} P_{1}$ & $3 p 3 d^{3} P_{2}$ & 12 & 21.846 & $6.27(3)$ & $1.61(-10)$ \\
\hline $3 p 2{ }^{1} D_{2}$ & $3 p 3 d^{3} F_{3}$ & & 42.509 & (4) & $-9)$ \\
\hline $3 p 2{ }^{3} P_{2}$ & $3 p 3 d^{3} D_{2}$ & 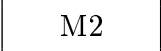 & 45.178 & 1.6 & $-9)$ \\
\hline $3 p 2{ }^{3} P_{2}$ & $3 p 3 d{ }^{1} F_{3}$ & 2 & 42.232 & (3) & $1.32(-9)$ \\
\hline $3 p 2{ }^{3} P_{1}$ & $3 p 3 d^{3} P_{1}$ & {$[2$} & 21.441 & $5.68(2)$ & $8.30(-12)$ \\
\hline $3 p 2{ }^{3} P_{1}$ & $3 p 3 d{ }^{1} D_{2}$ & 2 & 43.122 & 1.3 & $(-9)$ \\
\hline $3 p 2{ }^{1} D_{2}$ & $3 p 3 d d^{3} D_{3}$ & {$[2$} & 21.432 & $1.51(1)$ & $5.15(-13)$ \\
\hline $3 p 3 d{ }^{1} D_{2}$ & $3 d 2^{3} P_{1}$ & {$[2$} & 21.373 & $2.15(3)$ & $3.11(-11)$ \\
\hline $3 p 3 d{ }^{3} F_{2}$ & $3 d 2{ }^{3} F_{2}$ & 12 & 21.161 & $1.84(4)$ & $4.32(-10)$ \\
\hline $3 p 3 d{ }^{1} D_{2}$ & $3 d 2{ }^{3} P_{2}$ & 2 & 70 & & $(-11)$ \\
\hline $3 p 3 d{ }^{3} F_{3}$ & $3 d 2{ }^{3} F_{4}$ & 12 & 21.577 & 3) & $1.66(-10)$ \\
\hline $3 p 3 d^{3} F_{3}$ & $3 d 2{ }^{3} P_{2}$ & & 21.692 & $5.29(3)$ & $1.33(-10)$ \\
\hline $3 p 3 d{ }^{1} D_{2}$ & $3 d 2{ }^{3} F_{3}$ & 12 & 22.026 & (3) & $2.33(-10)$ \\
\hline $3 p 3 d^{3} F_{3}$ & $3 d 2{ }^{3} F_{3}$ & & 22.150 & & $5.66(-10)$ \\
\hline $3 p 3 d{ }^{3} P_{1}$ & & 2 & 22.495 & $1.05(4)$ & $2.94(-10)$ \\
\hline $3 p 3 d^{3} F_{4}$ & $3 d 2{ }^{1} G_{4}$ & & 41.410 & $2.25(4)$ & $7.11(-9)$ \\
\hline $3 p 3 d{ }^{3} D_{2}$ & $3 d 2{ }^{1} D_{2}$ & 12 & 41.708 & $4.63(3)$ & $8.28(-10)$ \\
\hline $3 p 3 d^{3} D_{1}$ & $3 d 2{ }^{3} P_{1}$ & & 42.854 & $1.22(4)$ & $1.43(-9)$ \\
\hline $3 p 3 d d^{3} D_{3}$ & & 12 & 43.174 & $1.31(4)$ & $4.69(-9)$ \\
\hline $3 p 3 d^{3} D_{1}$ & $3 d 2{ }^{3} P_{2}$ & {$[2$} & 43.626 & $2.10(2)$ & $4.32(-11)$ \\
\hline $3 p 3 d{ }^{3} P_{2}$ & $3 d 2{ }^{3} F_{3}$ & 42 & 43.848 & $2.89(3)$ & $8.42(-10)$ \\
\hline $3 p 3 d{ }^{1} F_{3}$ & $3 d 2{ }^{1} G_{4}$ & 12 & 46.167 & $1.45(3)$ & $6.35(-10)$ \\
\hline $3 p 3 d{ }^{1} P_{1}$ & $3 d 2{ }^{1} D_{2}$ & M2 & 46.659 & $9.26(3)$ & $2.32(-9)$ \\
\hline $3 p 3 d^{3} D_{3}$ & $3 d 2{ }^{3} P_{2}$ & M2 & 64.562 & $1.92(1)$ & $1.27(-11)$ \\
\hline $3 p 3 d{ }^{3} F_{4}$ & $3 d 2{ }^{3} F_{3}$ & M2 & 58.745 & $1.22(-1)$ & $8.51(-17)$ \\
\hline
\end{tabular}

In Table VII, we have presented results for wavelengths, transition probabilities and line strengths for forbidden transitions (E2, M1, and M2) among the lowest 132 levels of $\mathrm{W}^{62+}$. Transition parameters in the literature for the Mg-like tungsten are only available for a limited number of transitions. By applying the MCDHF code, wavelengths, transition probabilities and line strengths for E2, M1, and M2 transitions are here calculated by considering the electron correlation, QED and Breit contributions. Most of the transition data reported in the present work are new. We hope that these results will be helpful for the experimental and theoretical studies on the level structure of $\mathrm{W}^{62+}$.

\section{Conclusion}

In the present paper, the resonance and intercombination transitions of $n=3$ to $n=3$ are calculated using the MCDHF approach. The forbidden $3 l-3 l^{\prime}$ transitions for the $\mathrm{Mg}$-like ions are also investigated. QED corrections including screening and relaxation effects are also included. We have presented different results for fine structure energies from methods including different codes. The MCDHF gives excellent agreement with the RMBPT data and other theoretical results. It is found that the reliable treatments of the Breit and QED corrections play an important role in the correct assignment of different transitions and also in the accurate evaluation of atomic transition data for these high- $Z$ ions. However, theoretical accuracies are limited by the sizes of CI expansions, which also affected the open-shell systems that can be treated. Nevertheless, these computational constraints can be overcome with more efficient and compact basis sets as well as faster and more capable computers. The spectra present benchmark data for many yet unmeasured properties of $\mathrm{W}^{62+}$ and are particularly important in diagnostic of very high-temperature $L$-shell tungsten plasmas, and of future ITER plasmas.

\section{Acknowledgments}

This work was supported by the College of Science and Technology cooperation projects of Sanya under the Contract No. 2014YD2.

\section{References}

[1] T. Fan, C.R. Canizares, Astrophys. J. 539, 532 (2000).

[2] Y. Ralchenko, I.N. Draganic, J.N. Tan, J.D. Gillaspy, J.M. Pomeroy, J. Reader, U. Feldman, G.E. Holland, J. Phys. B At. Mol. Opt. Phys. 41, 021003 (2008).

[3] J.D. Gillaspy, I.N. Draganié, Y. Ralchenko, J. Reader, J.N. Tan, J.M. Pomeroy, S.M. Brewer, Phys. Rev. A 80, 010501(R) (2009).

[4] J. Clementson, P. Beiersdorfer, Phys. Rev. A 81 , 052509 (2010).

[5] J. Clementson, P. Beiersdorfer, G.V. Brown, M.F. Gu, H. Lundberg, Y. Podpaly, E. Träbert, Can. J. Phys. 89, 571 (2011).

[6] J.P. Marques, F. Parente, P. Indelicato, At. Data Nucl. Data Tables 55, 157 (1993).

[7] Y. Zou, C. Froese Fischer, J. Phys. B At. Mol. Opt. Phys. 34, 915 (2001).

[8] U.I. Safronova, A.S. Safronova, P. Beiersdorfer, J. Phys. B, At. Mol. Opt. Phys. 42, 165010 (2009).

[9] U.I. Safronova, A.S. Safronova, J. Phys. B At. Mol. Opt. Phys. 43, 074026 (2010).

[10] A.E. Kramida, T. Shirai, At. Data Nucl. Data Tables 95, 305 (2009).

[11] S.D. Loch, M.S. Pindzola, C.P. Ballance, D.C. Griffin, A.D. Whiteford, T. Pütterich, AIP Proc. 874, 233 (2006). 
[12] J. Clementson, P. Beiersdorfer, M.F. Gu, H.S. McLean, R.D. Wood, J. Phys. Conf. Ser. 130, 001204 (2008).

[13] C. Biedermann, R. Radtke, R. Seidel, T. Pütterich, Phys. Scr. T 134, 014026 (2009).

[14] C. Biedermann, R. Radtke, R. Seidel, E. Behar, J. Phys. Conf. Ser. 163, 014023 (2009).

[15] J. Reader, Phys. Scr. 134, 014023 (2009).

[16] T. Pütterich, R. Neu, R. Dux, A.D. Whiteford, M.G. ÓMullane, the ASDEX Upgrade Team, Plasma Phys. Control. Fusion. 50, 085016 (2008).

[17] A.E. Kramida, Can. J. Phys. 89, 551 (2011).

[18] J. Janagibayashi, T. Takano, A. Iwamae, H. Kubo, M. Hasuo, K. Itami, J. Phys. B At. Mol. Opt. Phys. 43, 144013 (2010).

[19] U. Feldman, J.E. Seely, E. Landi, Yu. Ralchenko, Nucl. Fusion 48, 045004 (2008).
[20] M.H. Chen, K.T. Cheng, Phys. Rev. A 84, 012513 (2011).

[21] G.C. Rodrigues, P. Indelicato, J.P. Santos, P. Patté, F. Parente, At. Data Nucl. Data Tables 86, 117 (2004).

[22] F. Hu, C. Wang, J. Yang, G. Jiangand, L. Hao, Phys. Scr. 84, 015302 (2011).

[23] L. Özdemír, G.G. Konan, S. Kabakci, Acta Phys. Pol. A 124, 649 (2013).

[24] P. Jönsson, X. He, C. Froese Fischer, I.P. Grant, Comput. Phys. Commun. 177, 597 (2007).

[25] I.P. Grant, Relativistic Quantum Theory of Atoms and Molecules, Springer, New York 2007.

[26] B.J. McKenzie, I.P. Grant, P.H. Norrington, Comput. Phys. Commun. 21, 233 (1980). 